\title{
An observer-inclusive hypothesis suggesting a reduction of the perceived universe to string interactions
}

\author{
Ehud Ahissar ${ }^{1}$ and Moshe Fried ${ }^{2}$ \\ ${ }^{1}$ The Department of Neurobiology, Weizmann Institute, Rehovot, Israel \\ ²Eye Research Institute, Tel Aviv University, Tel-Hashomer, Israel \\ Ehud.ahissar@weizmann.ac.il
}

\begin{abstract}
Physics and neuroscience share overlapping objectives, the major of which is probably the attempt to reduce the observed universe to a set of rules. The approaches are complementary, attempting to find a reduced description of the universe or of the observer, respectively. We propose here that combining the two approaches within an observer-inclusive physical scheme, bears significant advantages. In such a scheme, the same set of rules applies to the universe and its observers, and the two descriptions are entangled. We show here that analyzing special relativity in an observer-inclusive framework can resolve its contradiction with the observed non-locality of physical interactions. The contradiction is resolved by reducing the universe (including the observer) to a dynamic distribution of closed strings ("ceons") whose vibration waves travel at c. This ceons model is consistent with special and general relativity, non-locality and the holographic principle; it also eliminates Zeno's motion paradoxes. Yet, the model entails several new empirical predictions. Finally, the ceons model suggests a fundamental physical implementation of active biological perception. Paraphrasing Torricelli, this paper suggests that we live submerged in a $c$ of light.
\end{abstract}

\section{Summary and rational}

Relativity theory stems from the general principle of relativity, which asserts that the laws of physics should be the same for observers located in any reference body. Observers, in Einstein's relativity theory, are assumed to be "point observers," i.e., imaginary non-interacting sensors located in a dimensionless "point". Yet, relativity is based on measurements, or perceptions, of environmental variables such as the speed of light or simultaneity of events and, clearly, no measurement or perception can be implemented by ideal non-interacting "points". Crucially, interacting observers are themselves physical entities and thus should be included in the theory [1]. The current paper addresses this challenge by describing a physical model that includes the observer in it and is consistent with contemporary physics.

Our starting point is asking what makes the speed of light, c, a universal constant [2]. In other words, why isn't the speed of light emitted from a moving flashlight greater than that emitted from a stationary flashlight. We consider a time-measuring observer, a clock, and search for a scheme that is consistent 
with contemporary physics and in which the intrinsic frequency of the clock, i.e. its oscillating frequency as measured in a frame of reference moving with the clock, decreases with its speed in a Lorenzian manner. In one such scheme, the universe can be reduced to closed strings of various directions and radii, at all universal scales (from plank to cosmic sizes), whose vibration waves travel at c; such strings are termed here "ceons." In this model, and in line with de Broglie's wave interference, interactions between ceons produce everything we consider as matter and natural processes.

The observer-inclusion principle we add here is motion-interaction tradeoff. In a ceons-based universe, when the linear speed of a body increases, the energy of its internal ceon interactions decreases. In this case, Lorenz's time dilation emerges from a straightforward two-dimensional vectorial decomposition of the ceon's energy - decomposition into a motion axis (with c being the upper speed limit when no internal interactions occur) and an interaction axis, which determines the intrinsic frequency of the clock (or the aging rate of any body).

In this model, as the universe is reducible to interacting ceons, all speeds other than c observed by agents, including zero speed, are considered apparent speeds. Thus, a moving flashlight can be viewed in this scheme as sliding over ceons, rather than emitting ceons, and thus contributing nothing to their speed (in accordance with special relativity) although affecting their trajectories (which forms a new prediction).

We sketch here the proposed scheme in general terms, as much as is allowed by derivation from first principles by non-physicists, show its consistency with several major aspects of physics and list several empirical predictions. Importantly, being an observer-inclusive scheme our "sea of light" hypothesis is not about the universe 'by itself.' Rather, quoting Bohr, it is about what we can say about the universe [3].

\section{Introduction}

Einstein described the rationale leading to the theory of relativity as emerging from the question: "How does it come that certain reference-bodies (or their states of motion) are given priority over other reference-bodies (or their state of motion)?" Einstein stated that if no reason can be provided for this preference it should be assumed that the same laws of nature apply to all reference-bodies, a principle known as the general principle of relativity [2, p. 80-81]. On the same token one can ask: How does it come that certain speeds are given priority over other speeds? Namely, how does it come that only speeds $\leq \mathrm{c}$ can exist? Following Einstein's reasoning we ask for a reason for this preference and reason that this preference is justified only if an explanation exists. We have found a possible reason for this preference when we examined the physics of the observer, the clock in this case, and asked what would make its time measurement follow Lorentz' dilation.

The idea that a complete theory of the universe has to include the observers (including human observers) in it is not new [1]. In principle, one could imagine a non-interacting, 'ideal' observer, which observes 'the things in themselves,' assuming that they obey an observer-independent 'truth' (expressed for example in the form of mathematics [4]). In practice, however, it is not clear how would such an ideal observer get its initial hypotheses, verify its deductions, or share them with others. We thus assume here that observers are perceivers that interact with their environments, and whose ideas are deduced from such interactions. The physics we speak about is thus Bohr's physics, which "is not about reality, but about what we can say about reality," cited in [5].

The physics of fundamental particles revealed that all matter shares common building blocks. The current accepted 'standard' model describes a large set of possible elements, not all of them revealed so far [6]. Interestingly, however, it had been shown that at least some 'fundamental' particles can split 
to "sub components" in certain conditions [7]. Not surprisingly, then, several non-standard models had been suggested along the years, according to which the universe is composed of only one, or two, fundamental elements, whose various phases appear as the various 'standard' particles [8-14]. The most appealing single-element model is arguably the one described by the string theory $[14,15]$.

One principle all physicists agree upon is that there is no sharp physical border between bodies, or between a body and its environment. What appears to us as a body is a cluster of particles that tend to move together. Furthermore, particles are constantly moving and are continuously exchanged between bodies and between each body and its environment. Not only that, but a single particle, such as an electron, is described by a wave function which has no spatial limit $[16,17]$, and in principle can be localized anywhere in the entire universe [18]. Thus, although these characteristics do not affect the stochastic behavior of large bodies, due to the conservative distributions of their probabilities, they nevertheless demonstrate the inseparability of the universe to isolated components, beyond those of the most fundamental particles.

Not only that matter is inseparable, matter and energy are inseparable. These two forms of description are exchangeable. Such an inseparable universe was illustrated by Einstein as a giant mollusk, whose geometry determines what we call gravity [2]. But if this is the case, where does motion come from? If bodies are not separated, how can one body move relative to another [2]? Obviously, the only form of motion that is possible within such a mollusk is a change in local statistics, which immediately affects the rest of the mollusk. Also, it is clear that such a motion cannot be induced out of nothing; in one way or another it must be preserved within the mollusk through all times.

Motion is a trajectory in spacetime. Time measurements, such as the counting of periodic oscillations ('ticks') in an atomic clock or the aging of a body, are also trajectories in spacetime. Special relativity tells us that motion and time measures are fully coupled: whenever the rate of change over space (motion speed) increases the rate of change over time (e.g., clock tick frequency) decreases, and vice versa. This time-motion coupling conserves the spacetime interval. In our observer-inclusive framework, the conservation of the spacetime interval is explained by a conservation of the sum of internal interactions and body motion energies.

As we only speak from the perceiver's point of view, we must limit our description to that of the human's 'umwelt [19], i.e., the universe from the human's point of view. This description would be different from a description that may be expressed by rodents, ants or flies. Specifically, our perception and thus also our description of the world depends on the physiology of our senses and on the way they are operated $[20,21]$. One crucial such factor is that we can only sense changes in spacetime. Nevertheless, as we know, the human mind came up with the idea of stationary objects. Yet, as Zeno have elegantly shown, this idea cannot be straightforwardly reconciled with the idea of moving objects [22]. As we show here, Zeno's paradox exists only in the rational domain; it is resolved once a perceptual (i.e., observer-inclusive) theory of the universe is assumed.

In the following, we describe the major assumptions, propositions and corollaries of such a perceptual hypothesis of the universe, and derive several testable predictions. The rational and consequences of the hypothesis are discussed here in general terms, as much as is allowed by derivation from first principles by non-physicists.

\section{Assumptions of an observer-inclusive physical framework}

1. All observers (e.g., clocks, gauges, animals), obey the same physical laws as the rest of the universe.

2. All observations (measurements, perceptions) require interactions between physical elements. 


\section{The ceons hypothesis}

We describe our observer-inclusive hypothesis through a set of axioms and assumptions, their corollaries, and resulting propositions. The first proposition, which describes the emerging structural picture, may help visualizing our hypothesis. The validity of the proposed hypothesis should be judged by the internal consistency between its components and by their explanatory power with regard to natural phenomena. A self-consistent hypothesis, while not describing the 'things in themselves,' can be considered as a legitimate description of the human umwelt.

\section{Assumptions ("Axioms") of the ceons hypothesis:}

1. The universe can be reduced to closed strings whose vibration waves are travelling at $c$ (termed here "ceons", after $c$ ).

2. The ceons' radii range from Planck length to the cosmic scale $[23,24]$.

3. Following Bohr and others $[25,26]$ we assume that the fundamental invariants of all natural processes are interactions between ceons. We assume that every inter-ceon interaction is confined to a plane determined by the tangents of the two interacting ceons and thus can be decomposed to two orthogonal components, one directed along the equivalent vector of the interacting ceons and the other in an orthogonal direction within the "plane of interaction". The latter interaction components are termed here 'internal interactions'.

4. A body moving at a speed $<\mathrm{c}$ can be described as a collection of interacting ceons. For a body that is composed of $n$ ceons: the velocity vector is directed in the direction of the equivalent vector of all its ceons and its magnitude is given by

$$
\text { (1) } \quad v(n)=\frac{1}{n} \sum_{i=1}^{n} c \cos \theta_{i} \quad\left[\frac{m}{s}\right]
$$

where $\theta_{i}$ is the angle between the $i_{t h}$ ceon and the velocity vector within the "equivalent interaction plane" (i.e., the plan spanned by the equivalent vector and the tangent of the $i_{t h}$ ceon). The "intrinsic frequency" of that body ("intrinsic" here means: as observed in a frame of reference moving at $v$ ) is defined here as:

$$
\text { (2) } \quad f(n)=\frac{1}{n} \sum_{i=1}^{n} d\left|\sin \theta_{i}\right| \quad\left[\frac{1}{s}\right]
$$

where $d$ is the intrinsic frequency at rest $(v=0)$. 


\section{Corollaries:}

1. Interactions (from Axioms 1, 3 and 4). Interactions between ceons cause topology changes (e.g., $[27,28])$ and thus in essence change space. Changes can be translational (movement of the interaction zone along the velocity vector of the 'body' defined by the interactions, Fig. 1) or intrinsic to the 'body' (e.g., ticking of a clock or aging of a body). The range of feasible velocities of a given body is $|v| \leq c$ (Fig. 2).

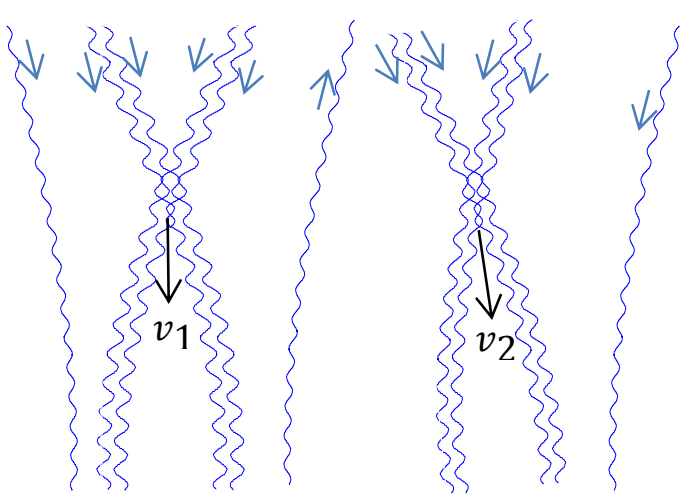

Figure 1. Illustration of ceons and group velocities within 2D space (a plane of interaction). Individual ceons (vibrating strings, blue) form 'bodies' by interactions with each other. The velocity of each body $\left(v_{1}, v_{2}\right)$ is determined by the group velocity of the interacting ceons. Space also contains noninteracting ceons.

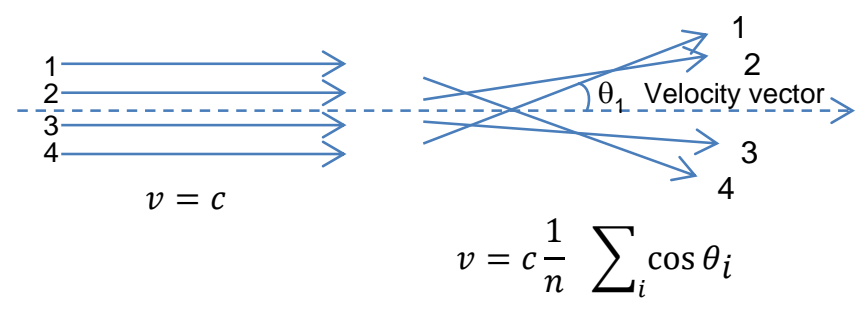

Figure 2. The velocity of a body $(v)$ is the vectorial sum of the velocity vectors of all its interacting ceons.

2. Vacuum (from Axioms 1, 2 and 3). A background density of ceon interactions is considered vacuum; densities above background level are considered matter and those below background level are considered anti-matter. Vacuum is thus full of ceons, which can be considered as Descartes' 'fine matter' [29] or Bohm's hidden paths [30] (see Corollary 10) and may account for the deduced vacuum energy [31].

3. Relativistic velocity (from Axioms 3 and 4). A clock moving at a velocity $v$ measures time according to the Lorenz-Einstein equation (assuming that the clock's rate is proportional to its intrinsic frequency):

$$
\frac{v^{2}}{c^{2}}+\frac{f^{2}}{d^{2}}=1
$$

where $f=\frac{1}{t}$ is the clock's rate and $d$ is the clock's rate at rest $(v=0)$ [32] (Appendix A1). Thus, as $v$ increases towards $c$ the clock's rate decreases towards 0 .

A universe obeying special relativity and the form of internal interactions proposed here (axioms 3 and 4) could not include strings whose wave speed is not c. Had there been strings with speed b, "beons", then there would exist clocks made of beons only, whose time counting stops when they move at $b$. If $b \neq c$ this would contradict special relativity. 
4. Space (from Axioms 1 and 2). Space and bodies reflect, and are nothing else than, ceon interactions. A body is a distortion of space density. This is a composition of Newton's and Einstein's descriptions: bodies are part of space (not 'floating' in an indifferent space; Einstein's point), but there is space without bodies (Newton's point) - the latter is the collection of all ceon fluxes with no interactions or with interactions whose density is not sufficient to interact with our senses through any existing device. This also means that a movement of a body is a change of space rather than a change over space [2]. The universe is composed of ceon interactions only - there is no underlying framework of any other kind.

5. Time (from Axioms 1 and 4). Time is a weighted count (or integration) of ceon interactions. When a body increases speed it means that more of its ceons travel in parallel and thus less interactions are counted [33], i.e., time slows down. Clocks, which are interaction counters, will report exactly that. In the theoretical limit, all ceons composing a body flow in the same direction and the body is said to travel at the speed of light. In this speed, no interactions occur between the ceons composing that mass (as they travel in parallel) and thus all processes, such as life and time counting, stop. The body becomes light.

6. Mass (from Axioms 1 and 2 and Corollary 2). Consistent with special relativity, a mass $(m)$ of a body reflects the total energy of ceon interactions composing it at a given moment. At a constant velocity this intensity, and thus the mass of the body, is proportional to the number of interacting ceons. Thus, a body traveling at $c$, such as a photon or a ray of light, has $m=0$. The apparent paradox implied by relativity theory, according to which the mass of a body that increases its velocity to c increases to infinity (while a photon's mass is 0 ) is solved here by the following simple consideration: bringing a body that is not moving at $c$ to $c$ requires that all its ceons will move in parallel. If the directions of the current ceons of the body are not changed, an infinite number of parallel ceons must be added. This explanation entails that simpler ways to bring a body to move at $c$ are by changing the directions of its ceons, or by replacing its ceons.

7. Acceleration (from Axioms 1,2 and 4). Acceleration is achieved by changing the relative composition of ceons trajectories in a body. For example, linear positive acceleration can be achieved by adding ceons that move in the accelerated direction or by changing ceons' trajectories. Similarly, rotations can involve addition of ceons or changes of ceons' trajectories.

8. Anti-matter and aether (from Axioms 1 and 2 and Corollary 2). Vacuum is defined as the background level of density of ceons interactions. Anti-matter can be defined as any decrement in interaction density below background density. When 'bodies' of matter and anti-matter meet, their interaction densities 'sum up'; if the resulting intensity equals that of background density then they annihilate each other, namely, they merge with the background. If not, they create matter or anti-matter of a new mass. Fluctuations of masses of bodies are thus expected to occur constantly.

9. The holographic principle (from Axioms 1 and 2 and Corollary 4). This principle states that the statistical description of a volume of space, in terms of entropy or information, is also encoded on a boundary to that volume [34, 35]. This is perfectly consistent with space being a flux of ceons - the externally-observable information contained in any volume of space is contained also in its boundary, as the boundary crosses all ceons that run through that volume.

10. Non locality (from Axioms 1 and 2 and Corollary 4). If ceons are strings, then there cannot be pure local actions in the universe; every action will affect a string whose wave may travel huge distances. As the wave's speed is $\mathrm{C}$, the effect of an action at point $A$ on an action occurring at point $B$ will be revealed only after the wave will make the distance from $B$ to $A$, although the change of the wave's trajectory was immediate. For example, in the dual slit experiment opening a new slit at point $A$ immediately changes the world by changing the trajectories of ceons, including those connecting to point $B$ at which a photon 
(which is a collection of ceons) is 'released' (i.e., its travelling waves are reset). The trajectories of the photon are thus changed immediately, while the effect is measurable only after a delay [30]. Even if the change was done after the photon was released, but before it reached point $A$, its trajectories will still be affected. The same principle holds for setting measurement devices on the path of entangled particles - they change the trajectories of ceons participating in these particles. Thus, the effect of an action can be considered non-local, although its measurement is limited by $\mathrm{c}$. In this sense, the ceons hypothesis provides a possible implementation of Bohmian non-local hidden variables [30].

11. Perception (from Axioms 1, 2 and 3 and Corollary 4). In a ceons-only universe, a body is a specific distribution of ceon interactions in spacetime. A body perceives information from its environment via ceon interactions connecting it with the environment. This temporary perceiver-perceived active bonding is apparent at macroscopic levels [21]. The ceons hypothesis further entails that at microscopic levels perception is active in the deep sense - it affects the perceived entity. When you look at the lethal quantum switch in Schrödinger's cat box you change the flow of ceons through that switch and thus affect it. In the same manner, in principle, you could feel when someone is looking at your back had you been sensitive enough.

12. Apparent speeds. We perceive speeds $<\mathrm{c}$. What makes $\mathrm{c}$, and not any other speed, a universal constant? The suggestion of the ceons model is that the only speed shared by all bodies at all times is the speed of their constituting elements, c. All other speeds depend on the current distribution of ceons, as determined by the ad-hoc perceiver-perceived tandem - these speeds are termed here "apparent speeds".

13. Zeno's paradoxes (from Axiom 1). The elimination of zero speed eliminates all Zeno's paradoxes - if there is no rest then there is no need to explain how (or when) a movement begins.

14. Quantum electrodynamics. The path integral principle states that the overall probability amplitude for a given event is equal to the integration of the probability amplitudes of all possible paths of the system in between the initial and final states, including those that are absurd by classical standards [36]. This is consistent with events reflecting interactions between ceons, where the collection of the paths of the ceons involved in an event form the set of all possible paths leading to that event.

\section{Propositions:}

1. The structure of the universe. The structure emerging from the axioms and corollaries described above is as follows. The universe is composed of strings of all feasible scales which vibrate at all feasible frequencies and interact with each other. Vibrations proceed along strings at c. String interactions are perceived by us as what we call matter and natural processes. String interactions may affect the characteristics of the interacting strings or of their interaction junction [28]. One such change is a change in the kinematics of the junction. A rule for a kinematic change that is consistent with special relativity is the one underlying the induction of a group velocity vector, as described in Axiom 4. With this rule, when the group velocity increases (the 'body' accelerates) the rate of ceon interactions in it decreases (corollary 3 and Appendix A1) such that a clock counts time slower and a body ages slower.

2. Energy. The only work done in the ceons universe is via ceon-ceon interactions. Energy is thus a measure of total ceon interactions. One can interpret the speed-time dependency as energy conservation: as more energy is invested in motion, less is invested in intrinsic interactions. Equation (3) thus reflects energy conservation: the sum of the energy invested in body motion $\left(\propto \frac{v^{2}}{c^{2}}\right)$ and that invested intrinsically $\left(\propto \frac{f^{2}}{d^{2}}\right)$ is constant.

3. Mass. At rest $(v=0) m c^{2}=E=h f=h d$. Thus, 


$$
m=\frac{h d}{c^{2}}
$$

where $d$ is the intrinsic frequency at rest and $\mathrm{h}$ is the Planck constant.

4. Force and inertial mass. Newton's second law, $F=m a$, can be reduced here to ceon counting. For example, in the case that acceleration involves addition of ceons (see Acceleration above) the magnitude of $a$ is the equivalent number of ceons added in the direction of $a$ (Axiom 4 ) in every unit of time divided by $m ; F$ is the equivalent number of ceons added in the direction of $a$ in every unit of time. The amount of added ceons should match the addition of mass due to the increase in velocity. A mass $m$ that is accelerated from rest to $v$, increases by a factor of $\left(1-\frac{v^{2}}{c^{2}}\right)^{-\frac{1}{2}}$ [37]. This factor should equal the factor by which the total intensity of ceon interactions increases.

A consistency check: We examine the simple case of adding 1 ceon to a mass $m$, composed of $n$ interacting ceons $(n>>1)$, that is at rest. $m$ is assumed to be proportional to $n$ and thus the new mass of the body, $m_{1}$, should be proportional to $n+1$. We'll verify this ratio by calculating the increased mass predicted by Tolman's equation. According to the ceons hypothesis, the new velocity of the body is $v_{l} \approx$ $v /(n+1)$ (see Appendix A1). Placing this equivalence in Tolman's equation leads to:

(4) $\frac{m_{1}}{m}=\frac{1}{\sqrt{1-\frac{v_{1}^{2}}{c^{2}}}}=\frac{1}{\sqrt{1-\frac{v^{2}}{c^{2}(n+1)^{2}}}}=\frac{n+1}{\sqrt{n^{2}+2 n+1-\frac{v^{2}}{c^{2}}}}$

and for $n>>1$ we get

Consistency approved.

$$
\frac{m_{1}}{m} \approx \frac{n+1}{n}
$$

Thus, the ceons hypothesis is consistent with relativistic mass changes as far as masses composed by many ceons are involved. As shown in Appendix A2, this probably includes all visible and measurable matter. Importantly, however, the ceons hypothesis may predict a deviation from Tolman's equation with very small masses. Quantifying such a deviation requires quantifying the total intensity of ceon interactions in a body (see Prediction 6).

5. Gravity. Gravity, according to the ceons hypothesis, merely reflects the statistics of ceons flow; the larger the mass the more ceons are flowing through it. There is no cause and effect here - gravity simply describes the universe. 'Attraction' between bodies is a mere reflection of the (biased) movement of the ceons via these bodies. For example, the motion of an apple towards Earth reflects the bias of ceons direction - more ceons are directed towards Earth than towards any other direction. This bias is because most ceons around Earth move through Earth. When the apple is held in place on its tree or on top of a hand, this bias is counter-balanced by an equivalent number of ceons moving up through it. Thus, as with Newton's second law (Proposition 4), gravity equation can also be reduced to ceon counting (Appendix A2). A reality check shows that the total number of ceons in the universe that is predicted by applying the ceons hypothesis to gravity is in the order of $10^{110}$, close to estimates of the number of subatomic particles that would fill all the space in the universe (Appendix A2). Another reality check shows that the "units" that would be assigned to a ceon by contemporary physics are $\left[\frac{\mathrm{m} \mathrm{kg}}{(\mathrm{m} / \mathrm{s})^{2}}\right]$, which are units of energy $(E)$ divided by the square of velocity (Appendix A2). Since the ceon's velocity is $c$, and a mass $m$ is proportional to the number of ceons, ceons' "units" are consistent with $E \propto m c^{2}$. 


\section{Corollaries (cont):}

15. The twin-clock 'paradox' (from Axioms 1 and 4, Corollary 3 and proposition 2). The twin paradox describes two identical clocks (or human twins) starting at rest $(v=0)$ at a given coordinates frame; one is then travelling away and back at $|v|>0$, meeting its stationary twin after completing the trip. According to special relativity, each clock should be delayed compared to the other by the same Lorentzian dilation interval (thus each twin should face an older brother). This apparent paradox is typically resolved outside special relativity, by considering accelerations - accelerations should age the travelling twin faster and compensate for velocity-driven dilation. According to the ceons hypothesis, however, there is no paradox as velocities can be discriminated along an absolute scale (see [38] and Prediction 1).

Note that empirical testing of the twin paradox is not possible (see Note in Prediction 1). Empirical tests of relativistic time dilation [39-41] are consistent with both relativity and the ceons hypothesis, as their time measurements are all done in one frame of reference (Earth or laboratory setting).

16. Galaxies dynamics. Several independent observations point to the fact that the visible mass in galaxies and galaxy clusters is insufficient to account for their dynamics, when analyzed using Newton's laws [42]. The ceons model is consistent with one explanation for this deviation, in which the gravitational field does not decay by $r^{-2}$ at large distances but rather gradually tends towards a decay by $r^{-1}[43,44]$. According to the ceons hypothesis, gravity reflects the flux of ceons moving through both the affected mass $(m)$ and the source mass $(M)$. As the distance from $M$ increases, more and more ceons leave the radiating flux as their trajectory curves out (recall that ceons are closed strings of all feasible radii). Thus, the number of ceons traveling towards $M$ through the envelope of a sphere of radius $r$ whose center is the center of $\left(M_{r}\right.$; Appendix 2$)$ decreases. The prediction is that it will decrease from $M_{r}=\frac{r^{2}}{G}$ to $M_{r} \propto \frac{r}{G}$, which would mean a transition from a three-dimensional to twodimensional expansion. Indeed, as distance increases, more ceons have a trajectory in directions that are tangential to the envelope rather than perpendicular to it. At the theoretical limit (at the 'edge' of the visible universe), all ceons move tangentially to the envelope and thus their number is proportional to $r$ rather than $r^{2}$.

\section{Critical predictions:}

1. Velocities can be discriminated along an absolute scale. The rate and/or intensity of all internal processes in a body should decrease as its velocity gets closer to that of light, judged in any frame of reference.

Note 1: This prediction contradicts the general principle of relativity [2]. Importantly, however, it should be noted that the application of the general principle of relativity to special relativity is untestable. Measuring a time interval requires two measurement times. In each measurement, the measuring and measured events must be simultaneous. As two inertial systems cross each other only once in spacetime, this dual simultaneity requirement cannot be satisfied [45]. In fact, if such dual measurements are forced, a self-contradiction is generated [46].

Note 2: Absolute velocity scale is also implicated by the absolute, or non-relativistic, characteristic of acceleration in general relativity. General relativity asserts that, if there is a change of relative velocity between different frames of reference, we can know in which frame the change occurred. Thus, theoretically, integrating along the world lines of any pair of frames of reference, starting from $t=0$ [47], allows the determination of their velocity, at any time $t>0$, along an absolute scale.

2. Acceleration and gravity are not equivalent. For an accelerating body $\Delta \boldsymbol{v}>\mathbf{0}$ and thus, according to the ceons hypothesis, $\Delta \boldsymbol{m}>\mathbf{0}$. Whereas for a stationary body in a gravitational field $\Delta \boldsymbol{v}=$ 
$\mathbf{0}$ and thus $\Delta \boldsymbol{m}=\mathbf{0}$ (see Proposition 3, "Force and inertial mass" above).

3. Curvilinear propagation in a gravitational field involves mass, energy and frequency changes. The decreased velocity of a beam of light traveling near a large mass should be accompanied by decreased mass, energy and frequency, as part of the ceons leave the beam (in favor of the big mass).

4. Mass should increase or decrease with velocity. In cases where ceons are added, mass will increase monotonically with velocity. In cases when only ceon trajectories are changed, mass will decrease monotonically with increasing velocities, because the intensity of interactions will decrease. An extreme case of the latter sort is an explosion - the total mass of the resulting matter should be smaller than the initial mass.

5. Mass should increase or decrease with heating. If ceons are added to the material under examination during heating its mass should increase. If ceons are not added, and thus only change trajectories that increase group motion speed, the mass should decrease.

6. Relativistic mass change should be revisited. The change of a mass of a body that is pushed (by adding ceons) to move at a velocity $v$ should deviate from Tolman's equation (see Eq. (4) above). This should be detectable with very small masses.

7. Perception is active at microscopic levels. Perceiving objects should change the trajectories of the ceons interacting in that object and thus modify its microscopic features. Quantitative predictions of such changes should await detailed physical-perceptual modeling.

\section{Difficulties in the ceons hypothesis:}

1. Speeds $>$ c. Granting physical elements the absolute speed c necessarily entails that relative speeds larger than $\mathrm{c}$ (up to $2 \mathrm{c}$ ) are feasible. In an observer-inclusive framework this does not necessarily contradicts special relativity. Although individual ceons can move at up to $2 \mathrm{c}$ relative to each other, these speeds cannot be measured as a single ceon cannot measure anything. Moreover, only bodies moving at $v<c$ can measure anything (via their internal interactions). As the amount of internal and cross-bodies interactions required for relative speed measurements are not known, one can assume consistency with special relativity by assuming that cross-body measurements can be manifested only with interactions that reduce the relative speed to $v<c$.

2. Gravitational attraction and the arrow of time. If gravity only describes the universe's geometry, then why bodies are always attracted and are never repelled from large masses such as Earth? In other words, why cannot we flip the direction of every single ceon in the universe and get bodies that escape from Earth? Another way to look at this issue is by asking what happens to the ceons running towards the Earth. If they all continue their trajectory through Earth, then while exiting Earth they should appear as being repelled from Earth.

There are two major hypothetical solutions here, both having implications on the directionality of natural process (the 'arrow of time'). There is no a-priori reason not to assume that both hypotheses are valid.

a. Mass expansion. Earth (and every celestial body) continuously grows in mass [48-50]. This, according to one hypothetical solution, occurs because part of the ceons entering Earth change their trajectory such that their diameters become smaller than that of Earth, as a result of their interactions with other ceons within Earth.

b. Geometry. Inward movement to Earth is more aligned towards Earth center than outward movement. That is, ceons moving towards Earth are more parallel to each other than those moving out from Earth. This hypothesis entails that the thought experiment in which ceons flip their direction 
cannot occur without the trajectories of these ceons being simultaneously changed. Such an alignment mechanism can be based on local interactions of ceons with gradients of mass density: If each ceon's wave is propagating towards maximal mass density at each point, ceons will be more aligned when moving towards a mass center than otherwise.

Both hypotheses suggest an irreversible process, which can account for the 'arrow of time', i.e., the time-asymmetry of macroscopic phenomena.

The geometrical hypothesis also accounts for the time dilation predicted by general relativity. As a body gets closer to a large mass, a larger fraction of its ceons move in parallel (towards that mass' center) and thus their internal interactions, within the body, are reduced and internal time measurement slows down.

\section{Discussion}

That the universe is composed of a single or dual common fundamental elements has been suggested more than once [8-13]. String theory presents the arguably most plausible description of such physics [14, 15], in which the universe, i.e., all forms of matter, including the so-called fundamental particles, can be reduced to dynamic distributions of one-dimensional open or closed vibrating strings. Closed strings possess two features which are crucial for the ceons hypothesis: their vibrations travel at $c$ along the string loop, and there is no physical limit for the size of the loop [14]. The ceons hypothesis assumes that strings exist in all possible radii, from Planck-size to universe-size [23].

In a way, the ceons hypothesis is an expansion of Bohr's model of the hydrogen atom to the entire universe. Instead of confining the circular motion of energy and mass to only very small (atoms) and very large (galaxies) scales, the ceons hypothesis suggests that everything in our universe moves in circles. The circular motion of vibrating energies entails a quantal universe: Closed strings cannot take every possible radius - only radii that allow an integer number of wavelengths can exist. The radii and wavelengths of course change continuously due to interactions, and some of these modulations may be directly observed. For example, modulations of ceons that surround Earth should result in frequencies corresponding to $c$ divided by the Earth perimeter - around 7-8 Hz. These frequencies are expressed as Schumann Resonances in the atmosphere [51], and are predominant in mammalian active perception [52].

Bohr was consistently arguing that physics is not about what is out there, but rather about what we can say about what is out there (or, equivalently, what we perceive as being out there) $[3,5,53]$. We could not agree more. Accordingly, our ceons model should be judged from the human observer perspective. As Bohr implied, our physics may merely reflect the common way we people perceive the world - our umwelt [19].

One common perception that requires explanation is the perception of stationary objects. What physics tells us is that everything is on the move. If you'd zoom into any 'stationary' object what you'd see are restless molecules in Brownian movements, in fluids, or lattice vibrations, in solids. Why don't we perceive the existence of these movements? This may be caused by our senses picking up the statistics of the environment and thus 'stationary' objects would mean stationary statistics [54]. Yet, this explanation would not suffice. We know for fact that not only the molecules in the environment and in our senses are constantly moving, but also that the entire organs collecting our sensations are constantly moving - a principle known as active sensing. As a result, there is hardly a single perceptual epoch during which the activation of sensory receptors is constant, even with 'stationary' objects [21, 55-57]. This indicates that the perception of steadiness does not emerge at the interaction between our body and the environment, but rather reflects a concept required for high-level functions such as 
planning or inter-subject information transfer.

An interesting implication of the ceons hypothesis is that the active component of sensing is not confined to the perceiving body but in fact also affects the perceived object. This is because the movements of the sensory organs change the trajectories of ceons connecting the perceiver and the object and thus actually affect the perceived object. Thus, reports such as 'I felt that someone is looking at me' may not be based merely on psychological cues but may also have a physical basis. This may also provide a mechanism accounting for the 'wave function collapse' idea in quantum mechanics [58]; the wave of a perceived (tiny) object may collapse to a single solution because a large fraction of its ceons are affected by the perceiver in a coherent way [59,60].

As stated above, this article is written by neuroscientists and attempts to bridge between neuroscience and physics. As such, it is based on a logical analysis of consistencies among currentlyexisting theories and observations in both fields. If our analysis is correct, then something like our ceons hypothesis is called for. Namely, the perceived universe should be reducible to interactions between ceon-like elements. The exact characteristics of these interactions, and the ways they may explain the entire spectrum of physical and perceptual phenomena, are of course beyond our capabilities and beyond the scope of this logical paper.

\section{Acknowledgments}

We thank Zohar Maliniak for igniting the spark for this endeavor, and Daniel Polani, Daniel Rohrlich, Roee Ozeri, Moti Milgrom, Sheldon Goldstein, Roger Penrose, Tom Kibble and Stanly Klein for helpful discussions and comments.

\section{References}

1. Leibniz, G.W., Système nouveau de la nature et de la communication des substances, aussi bien que l'union qu'il y entre l'âme et le corps. 1695, Hebrew translation Joseph Ur, 1969, Jerusalem: The Magnes Press The Hebrew University.

2. Einstein, A., Relativity: The special and the general theory. 15 ed. 1961, NY: Three Rivers Press.

3. $\quad$ Bricmont, J., Making sense of quantum mechanics. Vol. 37. 2016: Springer.

4. Penrose, R., et al., The large, the small and the human mind. 2000: Cambridge University Press.

5. Herbert, N., Quantum reality: Beyond the new physics. 1987: Anchor.

6. Braibant, S., G. Giacomelli, and M. Spurio, Particles and Fundamental Interactions: An Introduction to Particle Physics. 2012: Springer.

7. Kim, C., et al., Observation of spin-charge separation in one-dimensional srcu o 2. Physical review letters, 1996. 77(19): p. 4054.

8. Marsch, E., A model of leptons, quarks and bosons as composites of spinons. Arxiv preprint arXiv:0907.2538, 2009.

9. Harari, H., A schematic model of quarks and leptons. Physics Letters B, 1979. 86(1): p. 83-86.

10. Harari, H. and N. Seiberg, A dynamical theory for the rishon model. Physics Letters B, 1981. 98(4): p. 269-273.

11. Shupe, M.A., A composite model of leptons and quarks. Physics Letters B, 1979. 86(1): p. 8792.

12. Pati, J.C., A. Salam, and J. Strathdee, A Preon Model with Hidden Electric and Magnetic Type Charges. Nuclear Physics B, 1981. 185(2): p. 416-428.

13. Dehmelt, H., That I may know the inmost force that binds the world and guides its course. PHYSICA SCRIPTA-SUPPLEMENT T-, 1995. 59: p. 87-87. 
14. Greene, B., The Elegant Universe: Superstrings, Hidden Dimensions, and the Quest for the Ultimate Theory. 2000: Vintage Series, Random House Inc.

15. Witten, E., What every physicist should know about string theory, in Foundations of Mathematics and Physics One Century After Hilbert. 2018, Springer. p. 197-210.

16. Schrödinger, E., An undulatory theory of the mechanics of atoms and molecules. Physical Review, 1926. 28(6): p. 1049.

17. Born, M., Physical aspects of quantum mechanics. Nature, 1927. 119: p. 354-357.

18. Feynman, R.P., Space-time approach to non-relativistic quantum mechanics. Reviews of Modern Physics, 1948. 20(2): p. 367.

19. Uexkull, J.v., Theoretical biology. 1926, London: K. Paul, Trench, Trubner \& co. Itd.

20. Bergson, H., Creative evolution. 1983: University Press of America.

21. Ahissar, E. and E. Assa, Perception as a closed-loop convergence process. eLife, 2016. 5: p. e12830.

22. Lee, H.D.P., Zeno of Elea. 2015: Cambridge University Press.

23. Davis, A. and T. Kibble, Fundamental cosmic strings. Contemporary Physics, 2005. 46(5): p. 313-322.

24. Witten, E., Cosmic superstrings. Physics Letters B, 1985. 153(4-5): p. 243-246.

25. Bohr, N., Atomic Theory and the Description of Nature: 1. Four Essays, with an Introductory Survey. 1961: CUP Archive.

26. Mermin, N.D., What is quantum mechanics trying to tell us? arXiv preprint quant-ph/9801057, 1998.

27. Vilenkin, A. and E.P.S. Shellard, Cosmic strings and other topological defects. 2000: Cambridge University Press.

28. Copeland, E.J. and T. Kibble, Cosmic strings and superstrings. J Proceedings of the Royal Society A: Mathematical, Physical and Engineering Sciences, 2010. 466(2115): p. 623-657.

29. Dirac, P.A.M., Is there an aether? Nature, 1951. 168: p. 906-907.

30. Bohm, D., Hidden variables and the implicate order. Zygon®, 1985. 20(2): p. 111-124.

31. Copeland, E.J., M. Sami, and S. Tsujikawa, Dynamics of dark energy. J International Journal of Modern Physics D, 2006. 15(11): p. 1753-1935.

32. Einstein, A., On the electrodynamics of moving bodies. Annalen der Physik, 1905. 17(891): $p$. 50 .

33. Bailey, J., et al., Measurements of relativistic time dilatation for positive and negative muons in a circular orbit. Nature, 1977. 268(5618): p. 301-305.

34. Susskind, L. and E. Witten, The holographic bound in anti-de Sitter space. arXiv preprint hepth/9805114, 1998.

35. Bekenstein, J.D., Information in the holographic universe. Scientific American, 2003. 289(2): $p$. 58-65.

36. Feynman, R.P., QED: The Strange Theory of Light and Matter Princeton University Press. 1985, New Jersey: Princeton University press. p15.

37. Tolman, R.C., XXXIII. Non-Newtonian mechanics, the mass of a moving body. The London, Edinburgh, and Dublin Philosophical Magazine and Journal of Science, 1912. 23(135): p. 375380.

38. Tomaschitz, R., Cosmic time dilation: The clock paradox revisited. Chaos, Solitons \& Fractals, 2004. 20(4): p. 713-717.

39. Hafele, J. and R.E. Keating, Around-the-World Atomic Clocks: Predicted Relativistic Time Gains. Science, 1972. 177(4044): p. 166-168.

40. Hafele, J. and R.E. Keating, Around-the-World Atomic Clocks: Observed Relativistic Time Gains. Science, 1972. 177(4044): p. 168-170.

41. Chou, C.-W., et al., Optical clocks and relativity. Science, 2010. 329(5999): p. 1630-1633.

42. Faber, S.M. and J. Gallagher, Masses and mass-to-light ratios of galaxies. Annual review of astronomy and astrophysics, 1979. 17: p. 135-187.

43. Milgrom, M., A modification of the Newtonian dynamics as a possible alternative to the hidden mass hypothesis. The Astrophysical Journal, 1983. 270: p. 365-370.

44. Bekenstein, J. and M. Milgrom, Does the missing mass problem signal the breakdown of Newtonian gravity? The Astrophysical Journal, 1984. 286: p. 7-14. 
45. McCrea, W., Why the special theory of relativity is correct. Nature, 1967. 216(5111): p. 122.

46. Dingle, H., The case against special relativity. Nature, 1967. 216(5111): p. 119.

47. Hawking, S.W. The boundary conditions of the universe. in Astrophysical Cosmology Proceedings. 1982.

48. Tesla, N., Expanding Sun Will Explode Some Day, Tesla Predicts. NY Herald-Tribune, Aug, 1935. 18.

49. Dirac, P.A., The cosmological constants. Nature, 1937. 139: p. 323.

50. Carey, S.W., The expanding earth-an essay review. Earth-Science Reviews, 1975. 11(2): p. 105-143.

51. Rusov, V., et al., Can resonant oscillations of the earth ionosphere influence the human brain biorhythm? arXiv preprint arXiv, 2012: p. 1208.4970.

52. Kleinfeld, D., E. Ahissar, and M.E. Diamond, Active sensation: insights from the rodent vibrissa sensorimotor system. Curr Opin Neurobiol, 2006. 16(4): p. 435-44.

53. Snellman, H., 4 The Scientific Project: Knowledge Without Meaning? How Do We Know?: Understanding in Science and Theology, 2010. 6: p. 45.

54. Schrödinger, E., What is life?: With mind and matter and autobiographical sketches. 1992: Cambridge University Press.

55. Ahissar, E. and A. Arieli, Figuring space by time. Neuron, 2001. 32: p. 185-201.

56. Diamond, M.E., et al., 'Where' and 'what' in the whisker sensorimotor system. nature reviews neuroscience, 2008. 9(8): p. 601-12.

57. Rucci, M. and J.D. Victor, The unsteady eye: an information-processing stage, not a bug. Trends in Neurosciences, 2015. 38: p. 195-206.

58. Von Neumann, J., Mathematical foundations of quantum mechanics. 1955.

59. Ghirardi, G.C., A. Rimini, and T. Weber, Unified dynamics for microscopic and macroscopic systems. Physical Review D, 1986. 34(2): p. 470-491.

60. Penrose, R., The emperor's new mind: Concerning computers, minds, and the laws of physics. 1999: Oxford University Press. 


\section{Appendix A1. Special relativity}

We consider a physical clock travelling at velocity $v$ and measuring time ('ticking') at a rate of $f=\frac{1}{t}$. If $c$ is the speed of light in the void and $d$ is the rate of the clock at rest $(v=0)$, then Einstein-Lorentz equation for special relativity can be expressed as [32]:

$$
\frac{v^{2}}{c^{2}}+\frac{f^{2}}{d^{2}}=1
$$

\section{Consistency with the ceons hypothesis}

The magnitude of clock velocity is given by

$$
v(n)=\frac{1}{n} \sum_{i=1}^{n} c \cos \theta_{i}\left[\frac{m}{s}\right]
$$

and the clock's rate is given by:

$$
f(n)=\frac{1}{n} \sum_{i=1}^{n} d\left|\sin \theta_{i}\right|\left[\frac{1}{s}\right]
$$

where $n$ is the number of ceons composing the clock (see Axiom 4 above).

Lemma: The clock's velocity and rate obey the Einstein-Lorentz equation for any $n$ :

$$
\frac{v^{2}(n)}{c^{2}}+\frac{f^{2}(n)}{d^{2}}=1
$$

Where $v^{2}(n)$ and $f^{2}(n)$ indicate the squared velocity and frequency of a clock with $n$ ceons.

Comment: Using trigonometric identities of sums of sines and sums of cosines it can be shown that the lemma is correct for all bodies in which for every ceon moving at $\theta_{i}$ there exists a symmetric ceon moving at $-\theta_{\mathrm{i}}$. The following proof, if not mistaken, proves the lemma for any combination of ceons.

\section{Proof (by induction):}

(i) $\quad n=2$; for 2 ceons, which orient symmetrically around the velocity vector, each forming an angle of $\theta$ with the velocity vector, we get:

$$
\begin{array}{cc}
v=c \cos \theta & \frac{m}{s} \\
f=d|\sin \theta| & \frac{1}{s}
\end{array}
$$

and

$$
\frac{v^{2}(2)}{c^{2}}+\frac{f^{2}(2)}{d^{2}}=\cos ^{2} \theta+\sin ^{2} \theta=1
$$

(ii) Now we show that, for a given clock with $\mathrm{N}$ ceons, given that Eq. A1.1 is valid for $n$ it is also valid for $n+1$ for every $n<N$. That is, if

$$
\frac{v^{2}(n)}{c^{2}}+\frac{f^{2}(n)}{d^{2}}=1
$$

Then for every $n<N$

$$
\frac{v^{2}(n+1)}{c^{2}}+\frac{f^{2}(n+1)}{d^{2}}=1
$$


Induction proof:

$$
\begin{gathered}
v^{2}(n+1)=\frac{c^{2}}{(n+1)^{2}}\left(\sum_{i=1}^{n} \cos \theta_{i}+\cos \theta_{n+1}\right)^{2} \\
=\frac{c^{2}}{(n+1)^{2}}\left(\frac{n}{c} v(n)+\cos \theta_{n+1}\right)^{2} \\
v^{2}(n+1)=\frac{c^{2}}{(n+1)^{2}}\left(n^{2} \frac{v^{2}(n)}{c^{2}}+\cos ^{2} \theta_{n+1}+2 n \frac{v(n)}{c} \cos \theta_{n+1}\right)
\end{gathered}
$$

In the same fashion

$$
f^{2}(n+1)=\frac{d^{2}}{(n+1)^{2}}\left(n^{2} \frac{f^{2}(n)}{c^{2}}+\sin ^{2} \theta_{n+1}+2 n \frac{f(n)}{d}\left|\sin \theta_{n+1}\right|\right)
$$

Now

$$
\frac{v^{2}(n+1)}{c^{2}}+\frac{f^{2}(n+1)}{d^{2}}=\frac{1}{(n+1)^{2}}\left[n^{2}+1+2 n\left(\frac{v(n)}{c} \cos \theta_{n+1}+\frac{f(n)}{d}\left|\sin \theta_{n+1}\right|\right)\right]
$$

Thus,

$$
\frac{v^{2}(n+1)}{c^{2}}+\frac{f^{2}(n+1)}{d^{2}}=1
$$

if

(i) There exists two ceons in the clock for which Eq. A1.1 is satisfied, that is, which form symmetric angles with the axis of clock motion, and

(ii) for every $2 \leq n<N$ a ceon $n+1$ can be found in the clock such that

$$
\frac{v(n)}{c} \cos \theta_{n+1}+\frac{f(n)}{d}\left|\sin \theta_{n+1}\right|=1
$$

As Figure A1.1 shows, the solutions of Eq. A1.2 include the perimeter of a circle

$$
\frac{v^{2}(n)}{c^{2}}+\frac{f^{2}(n)}{d^{2}}=1
$$

Since this is assumed to hold true, condition (ii) is always satisfied.

We assume the condition (i) is also always satisfied, for all practical cases, and thus the lemma is proved. 


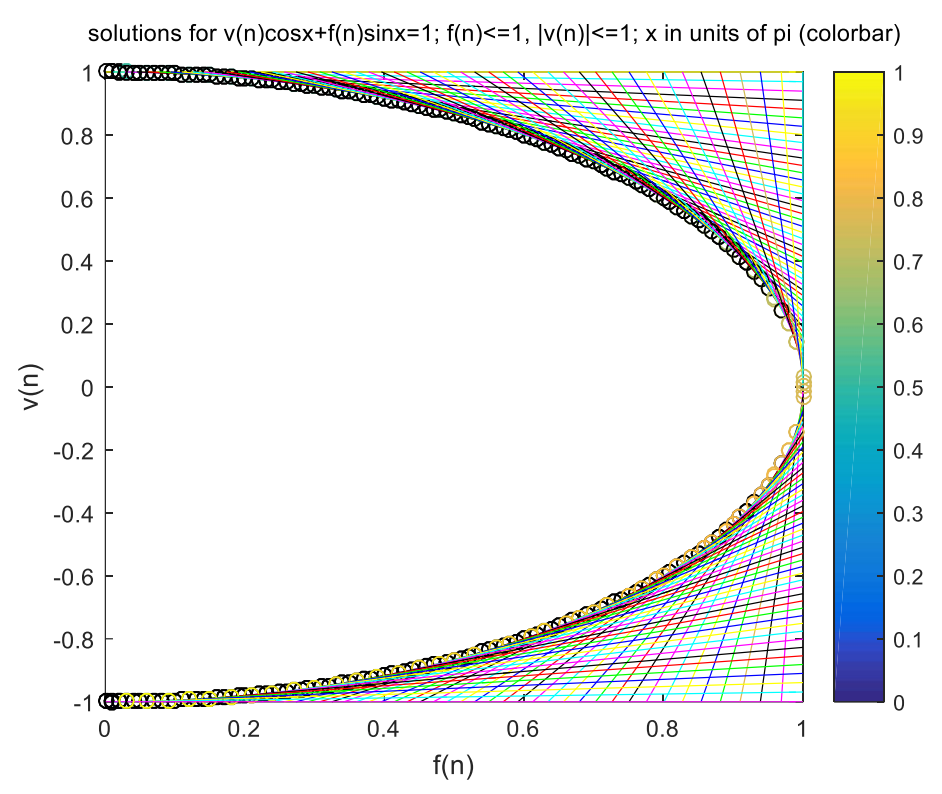

Figure A1.1. Solutions for Eq. A1.2. The color bar represents the values of $\theta_{n+1}$ in units of $\pi . v(n)$ and $f(n)$ are in units of $c$ and $d$, respectively.

\section{A1 Corollaries:}

1. At $v(n)=0, f(n)=d$ and thus only ceons whose $\left|\sin \theta_{i}\right|=1$ would satisfy Eq. 2 . As no axis of motion exists at $v=0, \theta_{i}$ is defined in relation to a point and thus its value is arbitrary. Thus, any pair of ceons with opposite wave velocities will preserve $v(n+2)=0$ and satisfy Eq. A1.2.

2. At $v(n)=c, f(n)=0$ and thus any ceon whose $\cos \theta_{i}=1$ would satisfy Eq. A1.2. This means that only ceons whose wave direction is the same as the direction of the body can be included in a body that moves at $c$, consistent with our assumptions.

\section{Appendix A2. Gravity as ceon geometry.}

\section{Ceon counting and the units of a ceon}

The gravity equation $F=\frac{G M m}{r^{2}}$ can also be reduced to ceon counting: $F$ is the number of ceons shared by $M$ and $m$. Each ceon traveling through $M$ has a probability of $\frac{m}{M_{r}}$ to go also through $m$, where $M_{r}$ is the number of ceons traveling towards $M$ through the envelope of a sphere of radius $r$ whose center is the center of $M$. Thus, $F=M \frac{m}{M_{r}}$ and thus $M_{r}=\frac{r^{2}}{G}$. Thus, according to the ceons hypothesis, $\mathrm{G}$ is a geometrical constant derived from the mean density of ceons in space. For a given direction, and a given time, the mean flux of ceons is

$$
S_{f}=\frac{M_{r}}{4 \pi r^{2}}=\frac{1}{4 \pi G}=1.210^{9}\left[\frac{\text { ceons }}{m^{2}}\right] \text {. }
$$

For this equation to be valid, the "units" of a ceon should be: $\left[\mathrm{m}^{2} /[G]\right]=\left[\mathrm{m}^{2} / \mathrm{m}^{3} \mathrm{~kg}^{-1} \mathrm{~s}^{-2}\right]=\left[\mathrm{kg} \mathrm{s}^{2} / \mathrm{m}\right]$. 
As ceons are the building blocks of everything else, their "units" describe relationships between various elements they build.

The "units" of a ceon $\left[\frac{\mathrm{m} \mathrm{kg}}{(\mathrm{m} / \mathrm{s})^{2}}\right]$ are units of energy $(E)$ divided by the square of velocity. Since the ceon's velocity is $\mathrm{c}$, and a mass $(\mathrm{m})$ is proportional to the number of ceons, ceons' "units" are consistent with $E \propto m c^{2}$.

\section{Reality check for the total number of ceons in the universe.}

The total number of ceons that travel at all times through $M$ equals the number of ceons crossing all possible envelopes around $M$ in a given time:

$S_{1}=\int_{0}^{\mathrm{R}} M_{r} \mathrm{dr}=\frac{1}{G} \int_{0}^{\mathrm{R}} \mathrm{r}^{2} \mathrm{dr}$

$S_{1}=\frac{R^{3}}{3 G}=5 * 10^{9} *\left(1.4 * 10^{26}\right)^{3}=1.4 * 10^{88}$ ceons

, where $\mathrm{R}$ is the radius of the visible universe (see also $\mathrm{http}: / /$ en.wikipedia.org/wiki/Observable universe;

http://mrob.com/pub/math/numbers-19.html).

The total number of ceons in the visible universe can thus be estimated as:

$S=S_{1} * \frac{N_{\text {stars }}}{N_{\text {ov }}}=\frac{1}{\mathrm{~N}_{\mathrm{ov}}} 1.4 * 10^{88} * 10^{23}=\frac{1}{\mathrm{~N}_{\mathrm{ov}}} 1.4 * 10^{111}$,

where $N_{o v}$ is the mean number of stars sharing a single ceon.

This estimate is intriguingly close to $10^{110}$, which is, according to one calculation, an estimate of the number of subatomic particles that it would take to fill all the space in the universe (see also http://mrob.com/pub/math/numbers-19.html).

The number of elementary particles in the visible universe, $N_{e p}$, is estimated between $10^{80} \div 10^{97}$ (see http://en.wikipedia.org/wiki/Elementary particle). The ratio between the estimated number of ceons and that of elementary particles $\left(D=\frac{S}{N_{e p}}=10^{14} \div 10^{31}\right)$ can provide information about the mean number of ceons composing an elementary particle.

For example, the mean number of ceons in a neutrino can be estimated as $S_{n}=\frac{10^{25}}{N_{o v}}$ and that in a photon as $S_{p}=\frac{10^{14}}{N_{o v}}$

Based on gravity and basic geometrical assumptions, we can assume that $1<\mathrm{N}_{\text {ov }} \ll 10^{23}$, where $10^{23}$ is the estimated number of stars in the universe, and based on the photon estimation above that $1<\mathrm{N}_{\mathrm{ov}}<10^{14}$. 